\title{
Elevated levels of pre $\beta 1$-high-density lipoprotein are associated with cholesterol ester transfer protein, the presence and severity of coronary artery disease
}

\author{
Xiao-min $\mathrm{Bu}^{\dagger}$, Dong-mei Niư ${ }^{\dagger}$, Jia Wu, Yun-long Yuan, Jia-xi Song ${ }^{*}$ and Jun-jun Wang ${ }^{*}$
}

\begin{abstract}
Background: Preß1-high-density lipoprotein (preß1-HDL), plays an important role in reverse cholesterol transport and exhibits potent risk for coronary artery disease (CAD). However, the association of plasma pre $\beta 1-H D L$ and cholesterol ester transfer protein (CETP) levels in CAD patients and the relationship of pre $\beta 1-\mathrm{HDL}$ with extent of CAD are debatable.

Methods: Preß1-HDL and CETP levels were measured by enzymed-linked immunosorbent assay (ELISAs) in 88 acute coronary syndromes (ACS), 79 stable coronary artery disease (SCAD) patients and 85 control subjects. The correlation analyses, multiple linear regression analyses and logistic regression analyses were performed, respectively.

Results: The preß1-HDL and CETP levels in ACS patients were significantly higher than those in SCAD patients and both of them were higher than controls'. Preß1-HDL levels were positively associated with CETP $(R=0.348, P=0.000)$, the diameter of stenosis $(R=0.253, P=0.005)$, the number of vessel disease $(R=0.274, P=0.002)$ and Gensini score $(R=0.227$, $P=0.009)$ in CAD patients. Stepwise multiple linear regression analyses showed that CETP was one of the determinants of preß1-HDL levels. Logistic regression analysis revealed that elevated preß1-HDL and CETP were potential risk factors for both ACS and SCAD.
\end{abstract}

Conclusion: The elevated preß1-HDL levels may change with CETP concentrations in CAD patients and were related to the presence and severity of CAD.

Keywords: Preß1-HDL, CETP, Coronary artery disease, Atherosclerosis

\section{Background}

Preß1-high-density lipoprotein (preß1-HDL), a quantitatively minor high-density lipoprotein (HDL), is formed as nascent apolipoprotein (apo) A-I enters plasma and as a substrate or product in interconversion of HDL species [1]. After receiving free cholesterol effluxed from ATP binding cassette transporter A1 (ABCA1) transporter, it is esterified with a free fatty acid derived from lecithin mediated by lecithin-cholesterol acyl transferase (LCAT) $[2,3]$. Cholesteryl esters (CE) are then incorporated into cores of $\alpha-\mathrm{HDL}$, subsuming pre $\beta 1-\mathrm{HDL}$. CE

\footnotetext{
* Correspondence: strive1225@126.com; wangjunjun9202@163.com ${ }^{\dagger}$ Equal contributors

Department of Clinical Laboratory, Jinling Hospital, School of Medicine, Nanjing University, 305East Zhongshan Rd., Nanjing 210002, China
}

are transferred from $\alpha$-high-density lipoprotein into cores of accepter lipoproteins by cholesterol ester transfer protein (CETP) [4]. The preß1-HDL was shown to be significantly correlated to the high efflux which was mediated by ABCA1 [5]. The fasting pre $\beta 1-\mathrm{HDL}$ concentration has been reported elevated in patients with coronary artery disease (CAD), hyperlipidemia, type 2 diabetic, obesity and hemodialysis [6-9]. It is proposed that increased pre $\beta 1-\mathrm{HDL}$ may be used as a marker of risk for structural CAD, myocardial infarction (MI) and cerebral vascular disease (CVD) [10]. However, a recent study reported that serum pre $\beta 1-\mathrm{HDL}$ levels detected by their new one-dimensional polyacrylamide gel electrophoresis (PAGE) system were negatively associated with severity of CAD [11]. This result needs further 
validition. Actually, the relationship between pre $\beta 1-\mathrm{HDL}$ and CAD is still controversial.

CETP, one of decisive factors that determines highdensity lipoprotein cholesterol (HDL-C), mediates the transfer of CE from HDL to low density lipoprotein (LDL), very low density lipoprotein (VLDL), and an exchange of triglyceride (TG). It relates to particle size, lipid composition and function of lipoprotein [12]. CETP also plays a key role in reverse cholesterol transport (RCT) and development of atherosclerosis [13, 14]. Several lines of evidence have shown that incubation of large $\alpha$-HDL particles with CETP could produce pre $\beta 1$ HDL $[6,7]$. which suggested the levels of pre $\beta 1-H D L$ may be associated with CETP. An earlier research performed in transgenic mice indicated that the expression of CETP resulted in an increase in the proportion of apoA-I in the pre $\beta 1-\mathrm{HDL}$ fraction and a stimulation of the efflux and esterification of cell-derived cholesterol [15]. However, study has been published to show the relationship of plasma pre $\beta 1-\mathrm{HDL}$ and CETP levels in CAD patients. Therefore, this study was undertaken to investigate the associations of plasma pre $\beta 1-\mathrm{HDL}$ and CETP in CAD patients, and to further elucidate the clinical values of pre $\beta 1$-HDL for evaluating the severity of CAD.

\section{Methods}

\section{Study subjects}

A total of 167 admitted CAD patients were randomly enrolled from the Department of Cardiology of Jinling Hospital between January 2014 and March 2015. All the patients were undergoing clinically indicated coronary angiography. Angiograms of all the CAD patients showed at least $50 \%$ stenosis in $\geq 1$ coronary artery. 88 patients with acute coronary syndromes (ACS) included acute myocardial infarction patients and unstable angina (UA) with Braunwald classification II or III, who exhibited the positive cardiac biomarkers result [cardiactroponin I $(\mathrm{cTnI})>0.090 \mathrm{ng} / \mathrm{ml}$, acute ischemic-type chest pain (lasting for $>15$ min, duration from symptoms onset to emergency admission within $72 \mathrm{~h}$ ) and characteristic electrocardiogram changes. 79 stable coronary artery disease (SCAD) patients with a normal electrocardiogram and documented normal left ventricular contractility, except for possible minor nonspecific ST-T features, had a minimum 1-year history without any cardiac events/procedures suggestive of ACS. The exclusion criteria of the CAD patients included mild disease of angiography (a stenosis of 10 to $50 \%$ of the luminal diameter in all the 3 coronary arteries), prior coronary revascularization, and the presence of renal disease. 85 control subjects selected from routine health examination were found normal in physical and electrocardiography and laboratory tests, and without diseases such as hyperlipidemia, hypertension, diabetes mellitus, or any clinical evident sign of atherosclerosis. In patients with ACS, blood samples were taken on admission. Blood samples were collected at least $12 \mathrm{~h}$ after fasting from control subjects and patients with SCAD. The blood sample was collected into EDTA $(1 \mathrm{mg} / \mathrm{ml})$ containing tube and plasma was promptly separated by a 15 min centrifugation at $3000 \mathrm{rpm}$, then stored at $-80{ }^{\circ} \mathrm{C}$ until analysis. This study protocol was approved by the Ethics Committee of Jinling Hospital (REC number: GH23335H) and all the subjects provided written informed consent.

\section{Angiographic analysis}

Catheterization was performed by either the Sones or the Judkins. Multiple views including angulated views were obtained, and the angiograms were evaluated. The extent of angiographically documented CAD was quantified in the left anterior descending coronary artery, the left circumflex artery, or the right coronary artery as follows: normal coronary arteries (smooth, with either no stenosis or a stenosis of $<10 \%$ of the luminal diameter), mild disease (a stenosis of 10 to $50 \%$ of the luminal diameter in one or more coronary arteries), or one-vessel, two-vessel, or three-vessel disease, defined as a stenosis of more than $50 \%$ of the luminal diameter in one, two, or three coronary arteries. To eliminate bias of judgment, angiographic observation and laboratory assays were conducted by different investigators in a double-blind way until all results were recorded and ready for statistical analysis. The severity of CAD was determined according to the Gensini score based on the degree of luminal stenosis and its geographical importance [16]. Reductions in the luminal diameter of $1-25 \%, 26-50 \%, 51-75 \%, 76-90 \%, 91-99 \%$ and total occlusion were respectively evaluated as 1, 2, 4, 8, 16 and 32. Each principal vascular segment was multiplied by a factor in accordance with the functional importance of the myocardial area supplied by that segment, that is, the left main $(\mathrm{LM})$ was assigned a multiplier $\times 5$; the proximal segment of the left anterior descending (LAD) and the proximal segment of the left circumflex $(\mathrm{LCX}) \times 2.5$; the middle of the LAD $\times 1.5$; the distal segment of the LAD, the right coronary artery $(\mathrm{RCA})$, the posterolateral artery, and the obtuse marginal artery $\times 1$; and other small vascular branches $\times 0.5$ [17].

\section{Assays}

Pre 1 1-HDL in human plasma was measured by a commercial preß1-HDL sandwich enzymed-linked immunosorbent assay (ELISA) kits from Sekisui Medical Co. LTD as described [18]. CETP was measured by sandwich ELISA as our laboratory described [19, 20]. TC, TG, high density lipoprotein (HDL) cholesterol were measured (Daiichi Pure Chemicals, Japan) on a Hitachi 7600 analyzer. Low-density lipoprotein cholesterol (LDL-C) was 
estimated using the Friedewald formula [21]. Creatine kinase-MB (CK-MB) was studied with Roche diagnostic kits in a COBAS C-8000 Roche autoanalyzer and studied by an immunological UV method, and cardiac troponin I (cTnI) tests were studied with TOSOH ST AIA-PACK cTnI 3-rd-Gen kit by AIA-2000ST TOSOH autoanalyzer.

\section{Statistic analysis}

Statistic analyses were performed with SPSS 16.0 and the values were expressed as the mean \pm standard deviation (SD). One Sample Kolmogorov-Smirnov Test was used to evaluate the normality of distribution of the variables. The skewed data, such as pre $\beta 1-\mathrm{HDL}, \mathrm{CETP}$ and TG concentrations were log-transformed to create a more normal distribution. The differences of variants among groups were analyzed by one-way analysis of variance (ANOVA), and the differences between groups were subsequently determined by Fisher LSD test when appropriate. The difference in the extent of angiographically documented disease, cTnI and CK-MB between two groups was analyzed by Student's $t$-test. The differences in sex among groups and medication were analyzed by the Chi-square test. Correlations between pre $\beta 1-\mathrm{HDL}$, CETP, Gensini score, cTnI and other variables in CAD patients were calculated by the non-parametric Spearman rank coefficient test. The stepwise multiple linear regression analyses $\left(P_{\text {in }}=0.05, P_{\text {out }}=0.10\right)$ were used to identify the influencing factor for plasma pre $\beta 1-H D L$ levels. The univariate and multivariate logistic regression analyses were used to calculate the approximation of the relative risk, odds ratio (OR) and 95\% confidence interval (CI) for selected variables. A two-tailed $P$-value less than 0.05 was considered statistically significant.

\section{Results}

Baseline clinical characteristics in the study group

The baseline clinical characteristics of the patients and control subjects, indications for coronary disease, and lipid measurements are showed in Table 1. There was no significant difference in age or sex among the ACS or SCAD patients and control subjects $(P>0.05) .54 .5 \%$ of ACS and $31.6 \%$ of SCAD patients received statin treatment in the present study at the time of sampling. The extent of angiographically documented CAD in the ACS patients was greater than that in SCAD patients (Table 1).

\section{Plasma pre $\beta 1-\mathrm{HDL}$, CETP and lipid concentrations in ACS and SCAD patients}

Compared with control subjects, preß1-HDL and CETP levels were found significantly increased in both patients with ACS and SCAD. Furthermore, preß1-HDL and CETP levels were significantly higher in ACS than those in SCAD (Table 2).
Table 1 Baseline clinical characteristic, lipid concentrations in the study groups

\begin{tabular}{|c|c|c|c|}
\hline Variable & $\begin{array}{l}\text { ACS } \\
(n=88)\end{array}$ & $\begin{array}{l}\text { SCAD } \\
(n=79)\end{array}$ & $\begin{array}{l}\text { Control } \\
(n=85)\end{array}$ \\
\hline Age (y) & $64.52 \pm 12.04$ & $67.42 \pm 13.34$ & $63.79 \pm 13.17$ \\
\hline Male/female & $62 / 26$ & $56 / 23$ & $64 / 21$ \\
\hline Hypertension (\%) & $59(67 \%)$ & $62(78 \%)$ & $0(0)$ \\
\hline Ischemic stroke (\%) & $32(36 \%)$ & $24(30 \%)$ & $0(0)$ \\
\hline Diabetes mellitus (\%) & 39 (44\%) & $30(38 \%)$ & $0(0)$ \\
\hline \multicolumn{4}{|l|}{ Medication } \\
\hline Statins, n (\%) & $48(54.5)$ & $25(31.6)$ & $0(0)$ \\
\hline ACEI/ARB, n (\%) & $63(71.6)$ & $51(64.6)$ & $0(0)$ \\
\hline Beta blockers, n (\%) & $58(65.9)$ & $42(53.2)$ & $0(0)$ \\
\hline Aspirin (\%) & $60(68.2)$ & $26.2(21.0)$ & $0(0)$ \\
\hline \multicolumn{4}{|l|}{ Angiographic analysis } \\
\hline Maximal stenosis (\%) & $89.34 \pm 15.62^{* *}$ & 23.87 & - \\
\hline $\begin{array}{l}\text { Number of vessel } \\
\text { disease }\end{array}$ & $2.04 \pm 0.85$ & $1.14 \pm 0.93$ & - \\
\hline cTnl, ng/L & $19.42 \pm 71.19^{* *}$ & $0.03 \pm 0.02$ & - \\
\hline CK-MB, U/L & $75.42 \pm 126.14^{* *}$ & $11.68 \pm 4.12$ & \\
\hline Gensini score & $62.23 \pm 41.58^{* *}$ & $17.20 \pm 18.39$ & - \\
\hline
\end{tabular}

Data are presented as the mean \pm SD or number (\%) of subjects Compared with SCAD: ${ }^{*} P<0.05,{ }^{* *} P<0.01$

\section{Associations among pre $\beta 1-\mathrm{HDL}, \mathrm{CETP}$, other lipid levels and the extent of CAD}

To study the relationship of preß1-HDL, CETP, other lipid parameters and the extent of CAD, Spearman rank correlation analyses were performed. In all the CAD patients, including ACS and SCAD patients, pre $\beta 1-\mathrm{HDL}$ were found positively correlated with CETP $(r=0.348, P=0.000)$, maximal stenosis $(r=0.253, P=0.005)$, number of vessel disease $(r=0.274, P=0.002)$, Gensini score $(r=0.227, P$ $=0.009)$, TC $(r=0.401, P=0.000)$, TG $(r=0.195, P=0.017)$ and LDL-C $(r=0.309, P=0.000)$; and CETP were positively correlated with number of vessel disease $(r=0.238$, $P=0.013)$, Gensini score $(r=0.282, P=0.002)$, TC $(r=0.209$, $P=0.016)$ and LDL-C $(r=0.202, P=0.023)$ (Table 3$)$.

Table 2 Plasma preß1-HDL, CETP and other lipid concentrations in ACS, SCAD and control groups

\begin{tabular}{llcl}
\hline Variable & $\begin{array}{l}\text { ACS } \\
(n=88)\end{array}$ & $\begin{array}{l}\text { SCAD } \\
(n=79)\end{array}$ & $\begin{array}{l}\text { Control } \\
(n=85)\end{array}$ \\
\hline $\mathrm{TC}(\mathrm{mmol} / \mathrm{L})$ & $4.68 \pm 1.34$ & $4.16 \pm 1.10$ & $4.62 \pm 0.58$ \\
$\mathrm{TG}(\mathrm{mmol} / \mathrm{L})$ & $2.04 \pm 1.47^{* *}$ & $1.70 \pm 1.31^{* *}$ & $1.09 \pm 0.41$ \\
$\mathrm{HDL}-\mathrm{C}(\mathrm{mmol} / \mathrm{L})$ & $0.99 \pm 0.23^{* *}$ & $1.13 \pm 0.72^{* *}$ & $1.31 \pm 0.25$ \\
$\mathrm{LDL}-\mathrm{C}(\mathrm{mmol} / \mathrm{L})$ & $2.91 \pm 1.02^{*}$ & $2.88 \pm 0.85^{*}$ & $2.45 \pm 0.81$ \\
$\operatorname{Pre} \beta 1-\mathrm{HDL}(\mu \mathrm{g} / \mathrm{L})$ & $36.24 \pm 17.03^{* *, \#}$ & $24.68 \pm 21.02^{* *}$ & $7.44 \pm 5.49$ \\
CETP $(\mathrm{mg} / \mathrm{L})$ & $3.42 \pm 1.80^{* *, \# \#}$ & $2.05 \pm 1.10^{*}$ & $1.52 \pm 0.98$ \\
\hline
\end{tabular}

Compared with control: ${ }^{*} P<0.05{ }^{* *} P<0.01$

Compared with SCAD: ${ }^{\#} P<0.05 ;{ }^{\# \#} P<0.01$ 
Table 3 Spearman rank correlations between pre $\beta 1-H D L, C E T P$ and other variable in CAD patients

\begin{tabular}{lll}
\hline Variable & Pre $\beta 1-\mathrm{HDL}$ & CETP \\
\hline TC & $0.401^{* *}(P=0.000)$ & $0.209^{*}(P=0.016)$ \\
TG & $0.195^{*}(P=0.017)$ & $0.126(P=0.150)$ \\
HDL-C & $0.052(P=0.537)$ & $-0.0335(P=0.692)$ \\
LDL-C & $0.309^{* *}(P=0.000)$ & $0.202^{*}(P=0.023)$ \\
CETP & $0.348^{* *}(P=0.000)$ & - \\
Preß1-HDL & - & $0.348^{* *}(P=0.000)$ \\
Maximal stenosis(\%) & $0.253^{* *}(P=0.005)$ & $0.087(P=0.370)$ \\
Number of vessel disease & $0.274^{* *}(P=0.002)$ & $0.238^{*}(P=0.013)$ \\
Gensini score & $0.227^{* *}(P=0.009)$ & $0.282^{* *}(P=0.002)$ \\
\hline${ }^{*}{ }^{*}{ }^{* *} P<0.01$ & &
\end{tabular}

${ }^{*} P<0.05 ;{ }^{* *} P<0.01$

To further explore the possible factors affecting pre $\beta 1$ HDL levels in CAD patients, the multiple linear regression analyses was performed. Consequently, the TC, CETP accounted for $19.9 \%$ of the variation of pre $\beta 1$ HDL levels, when all variables that were significantly correlated with pre $\beta 1$-HDLwere included as independent variables (Table 4 ).

\section{Preß1-HDL and CETP as risk factors for ACS and SCAD}

The univariate and multivariate logistic regression analyses were next performed to evaluate the possible associations of pre $\beta 1-\mathrm{HDL}$ and CETP with CAD. As shown in Table 5, using the control group as the reference category when the ACS, SCAD or control group was treated as a dependent three-category variable, the univariate analyses revealed that increased pre $\beta 1-\mathrm{HDL}$ or CETP was the potential risk factor for ACS (pre $\beta 1-\mathrm{HDL}, \mathrm{OR}=1.161$, 95\% CI: $1.107-1.219, P<0.001$; CETP, OR $=3.055,95 \%$ CI: 2.158-4.326, $P<0.001$ ), and for SCAD (preß1HDL, OR1.142, 95\% CI: 1.089-1.198, $P<0.001$; CETP, $\mathrm{OR}=1.632$, 95\% CI: $1.188-2.217, P<0.05)$. To further

Table 4 Stepwise multiple linear regression analyses of factors affecting preß1-HDL levels in CAD patients

\begin{tabular}{ccl}
\hline Unstandardized coefficients & $\begin{array}{l}\text { Standardized } \\
\text { coefficient }\end{array}$ \\
\cline { 2 - 3 } & B value \\
B & SE & \\
\hline
\end{tabular}

Independent variables in the model

Constant

$\begin{array}{lllll}\text { TC } & 8.448 & 1.917 & 0.371 & 0.000 \\ \text { CETP } & 2.706 & 1.216 & 0.187 & 0.028\end{array}$

Independent variables excluded from model

$\begin{array}{lll}\mathrm{TG}^{\mathrm{a}} & 0.086 & 0.354\end{array}$

LDL-C $C^{\mathrm{a}}$

$-0.045$

0.743

The dependent variable was pre $\beta 1$-HDL (adjusted $\mathrm{R}^{2}=0.199$ ). TC, TG, LDL-C, Ox-LDL and CETP were used for independent variables

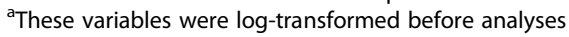

Table 5 Logistic regression analysis of risk factors for ACS and SCAD

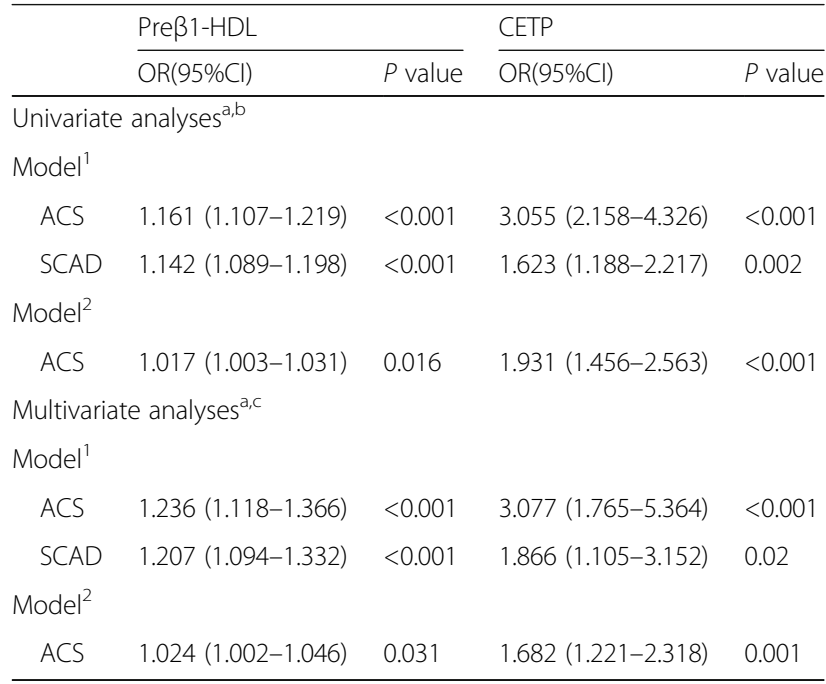

Model $^{1}$ : the reference category was the control group

Model $^{2}$ : the reference category was the SCAD group

${ }^{\mathrm{a}}$ In univariate and multivariate logistic regression analyses, the ACS, SCAD or control group was treated as a dependent three-category variable and the $\mathrm{OR}$ was considered statistically significant when the lower limit of the $95 \% \mathrm{Cl}$ was $>1.0$ and the $P$-value was $<0.05$

bonly one of Pre $\beta 1-H D L, C E T P$ was included in the univariate analyses

'The age, gender and coexisting conditions were adjusted in the multivariate analyses

evaluate the associations of pre $\beta 1-\mathrm{HDL}$ and CETP with the severity of CAD in ACS or SCAD patients, pre $\beta 1$ HDL $(\mathrm{OR}=1.017,95 \% \mathrm{CI}: 1.003-1.031, P=0.016)$ and CETP $\quad(\mathrm{OR}=1.931, \quad 95 \% \mathrm{CI}: \quad 1.456-2.563, \quad P<0.001)$ were found closely associated with ACS group when treated the SCAD group as the reference category.

After adjusting for age, gender and plasma lipid levels and using the control group as the reference category, when the ACS, SCAD or control group was treated as a dependent three-category variable, the increased pre $\beta 1-H D L$ or CETP was also revealed to be the potential risk factor for both ACS (pre $\beta 1-\mathrm{HDL}, \mathrm{OR}=1.236$, 95\% CI: 1.118-1.366, $P<0.001$; CETP, OR $=3.077,95 \%$ CI: $1.765-5.364, P<0.001$ ) and SCAD (pre $\beta 1-H D L$, $\mathrm{OR}=1.207, \quad 95 \%$ CI: $1.094-1.332, \quad P<0.001$; CETP, $\mathrm{OR}=1.866,95 \% \mathrm{CI}: 1.105-3.152, P=0.020)$. Similarly, pre $\beta 1$-HDL or CETP was also showed closely association with ACS group when treated the SCAD group as the reference category.

\section{Discussion}

This study for the first time shows that the elevated pre $\beta 1-H D L$ levels were related to the presence and severity of CAD and changed with CETP levels in CAD patients. Compared with control subjects, the plasma levels of preß1-HDL and CETP were significantly increased in both SCAD and ACS patients. CETP was 
found to be one of potential predictors for pre $\beta 1-\mathrm{HDL}$ levels. Furthermore, the pre $\beta 1-\mathrm{HDL}$ levels in ACS patients were significantly higher than those in SCAD patients, and were significant risk for both ACS and SCAD patients.

Pre $\beta 1-\mathrm{HDL}$ has been reported to be increased in CAD patients [10]. Amar et al. found that preß1-HDL concentrations were 2-fold higher in individuals with ischemic heart disease (IHD) than no IHD groups [22]. Besides, it has been reported that preß1-HDL level was markedly higher in the unstable angina pectoris (UAP) subgroup than in the stable angina pectoris (SAP) subgroup [23, 24]. In this study, we found that the pre $\beta 1$ HDL levels increased in CAD patients and it's levels in ACS patients were significantly higher than those in SCAD patients, which was consistent with earlier observations $[23,24]$ suggesting that the increased pre $\beta 1$ HDL concentrations might be one of the major factors for the occurrence and severity of CAD. In addition, we further found that plasma preß1-HDL was positively related with degree of coronary artery stenosis, number of vessel disease and Gensini score, suggesting that the increased pre $\beta 1-\mathrm{HDL}$ may associated with cholesterol accumulation in coronary artery vessels. Miyazaki et al. [25] and Sethi et al. [22] also found that the preß1-HDL level was high in hyperlipidemic and ischemic heart disease subjects. In our and the above studies, pre $\beta 1$ HDL level was measured by ELISA using anti-human preß1-HDL monoclonal antibody (MAb 55201) specifically reacted with apoA-I associated with pre $\beta 1-\mathrm{HDL}$ in plasma. Recently, Chen et al. described that serum pre $\beta 1-H D L$ levels were decreased and negatively associated with Gensini score in patients with coronary artery diseases [11], which was inconsistent with the above and previous studies [10, 23, 24]. Chen et al. isolated the pre $\beta 1-\mathrm{HDL}$ using a native polyacrylamide gel electrophoresis (PAGE) system with polyclonal anti-human apoA-I and detected the levels of preß1-HDL by densitometry, which is a semi-quantitative method. Whereas, ELISA is a quantitative method. Although significantly high pre $\beta 1-H D L$ levels were observed in CAD cases, the association between preß1-HDL level and CAD prevalence was inconsistent. Bela et al. reported that the increased pre $\beta 1-H D L$ was not significantly associated with CAD prevalence but associated with recurrent CAD events [26, 27]. A large well-characterized clinical cohort study confirmed the association between increased levels of pre $\beta 1-\mathrm{HDL}$ and risk of CAD, and proposed that high levels of preß1-HDL remained a significant predictor of CAD even after adjustment for traditional risk factors [10].

Pre $\beta 1-H D L$ is considered to be an atherogenic lipoprotein; although Lynnda et al. found that pre $\beta$-HDL formation was affected by high-normal free thyroxine in type 2 diabetes mellitus [28]. The mechanisms by which it contributes to atherosclerosis remain widely unknown. It is important to realize that the RCT pathway is a cyclical way $[10,29,30]$. The increase of preß1-HDL may be indicative of a defect in one part of the pathway. It has been reported that the causes of increased preß1-HDL included the defect of ABCA1 transporter, low levels of LCAT, overexpression of phospholipid transfer protein (PLTP) or increased plasma cholesterol efflux capacity from macrophages [3, 31-33]. Increasing evidence suggested that the concentration and composition of plasma lipids and lipoproteins were markedly affected by the acute phase reactions, which associated with injury, inflammation or sepsis [34]. The changes of the concentration and composition of plasma HDL in patients with ACS could be secondary to systemic acute phase response. As an acute phase protein mainly produced in the liver, levels of serum amyloid A (SAA) both in plasma and HDL compositions increase markedly in inflammation [34]. It has been suggested that these changes in HDL apoprotein pattern could increase HDL catabolism, impair reverse cholesterol transport. This would reverse the flux of cholesterol between macrophages and HDL and result in increased macrophage uptake. Therefore, the changes of the composition of plasma HDL in patients with ACS that secondary to systemic acute phase response were proinflammatory. Pre $\beta 1-\mathrm{HDL}$, as a minor HDL, may be secondary to systemic acute phase response in ACS [35]. Future research should focus on the role of pre $\beta 1-\mathrm{HDL}$ in this process.

Several lines of evidence suggested that preß1-HDL concentration associated with CETP level [36]. When plasma was incubated with exogenous CETP at $37{ }^{\circ} \mathrm{C}$, total preß1-HDL concentration increased [37]. In cholesterol-fed rabbits, serum pre $\beta 1$-HDL concentration and CETP activity were 32 and $33 \%$ higher than those in control rabbits respectively [38]. And the plasma preß1-HDL concentration was positively correlated with the hepatic CETP mRNA level [38]. In a human study, probucol increased CETP mass by $20 \%$, and maintained a high preß1-HDL concentration in hypercholesterolemics [39]. However, Nicholls et al. found that evacetrapib, potent CETP inhibitor significantly increased preß1-HDL levels in dyslipidemic patients [40]. Our present results further confirmed that the plasma pre $31-H D L$ levels elevated with high CETP mass in patients with CAD. As we all known, CETP plays an important role in the transfer and exchange of CE and TG among lipoproteins, so that it affects lipid composition, concentration, particle size and function of lipoprotein [12]. In addition, it is now accepted that virtually all of the efflux mediated by the ABCA1 transporter goes to pre $\beta 1-H D L$, which contains only one molecular species 
of protein, apoA-I. Then their unesterified cholesterol is esterified by the action of LCAT, and subsumed into larger cholesteryl ester-rich HDL particles from which the esters can be transferred to acceptor lipoproteins, including VLDL and LDL, mediated by CETP. Thus, the present results indicated that in CAD patients, with the increasing CETP concentration, more pre $\beta 1-\mathrm{HDL}$ were regenerated during the CETP-mediated transfer process. The current study supports the concept that incubation of large $\alpha-\mathrm{HDL}$ particles with CETP may produce pre $\beta 1-\mathrm{HDL}$, and increased pre $\beta 1-\mathrm{HDL}$ may be an indicator in the reverse cholesterol transport pathway, which may be closely related to the presence of CAD.

The limitations of this study include the fact that the control subjects were not examined by coronary angiography to exclude potential CAD. This is mainly limited by the wishes of the individual volunteers; however, we have performed physical, electrocardiography and laboratory tests for the control subjects and make sure that all of the controls are without diseases such as hyperlipidemia, hypertension, diabetes mellitus, or any clinical sign of atherosclerosis. In addition, subjects using statin were not excluded from the present study, fortunately, statin treatment decreases pre $\beta 1-\mathrm{HDL}$ so that it can reveal better the increase of pre $\beta 1-\mathrm{HDL}$ in CAD [40, 41]. Besides, future studies should directly capture and analyze the embolized debris for preß1HDL and CETP.

\section{Conclusions}

The present study demonstrated that elevated plasma concentrations of preß1-HDL reflect the presence and extent of angiographically documented CAD, especially clinically expressed in ACS. The plasma pre $\beta 1-\mathrm{HDL}$ levels were potently correlated with CETP in CAD, and may change with CETP concentrations. These finding will contribute to the understanding of the pathogenetic role of preß1-HDL and CETP in CAD. Further studies are needed to validate these associations and to elucidate pathophysiologic mechanisms of plasma preß1-HDL in CAD.

\footnotetext{
Abbreviations

ABCA1: ATP binding cassette transporter A1; ACS: Acute coronary syndromes; ANOVA: One-way analysis of variance; apo: Apolipoprotein; CAD: Coronary artery disease; CE: Cholesteryl ester; CETP: Cholesterol ester transfer protein; $\mathrm{Cl}$ : Confidence interval; CK-MB: Creatine kinase-MB; CTnl: Cardiac troponin I; CVD: Cerebral vascular disease; ELISA: Enzymed-linked immunosorbent assay; HDL: High-density lipoprotein; HDL-C: High-density lipoprotein cholesterol; IHD: Ischemic heart disease; LAD: Left anterior descending; LCAT: Lecithincholesterol acyl transferase; LCX: Left circumflex; LDL: Low-density lipoprotein; LDL-C: Low-density lipoprotein cholesterol; LM: The left main; MI: Myocardial infarction; OR: Odds ratio; PAGE: Polyacrylamide gel electrophoresis; PLTP: Phospholipid transfer protein; preß1-HDL: Preß1-high-density lipoprotein; RCT: Reverse cholesterol transport; SAP: Stable angina pectoris; SCAD: Stable coronary artery disease; SD: Standard deviation;
}

TC: Total cholesterol; TG: Triglyceride; UAP: Unstable angina pectoris; VLDL: Very low density lipoprotein

\section{Acknowledgements \\ Not applicable.}

\section{Funding}

This study was generously funded by the National Natural Science Foundation of China (No. 81271904, 81401742, 81572073 and 81572074), the Special-funded Program on National Key Scientific Instruments and Equipment Development of China (2012YQ030261).

\section{Availability of data and materials}

All data generated or analyzed during the current study are available from the corresponding author on reasonable request.

\section{Authors' contributions}

JJW and JXS designed the study. XMB, DMN and JXS collected the data. JXS, $X M B, D M N, J W$ and YLY did the data analysis and interpretation. XMB, JXS and JJW wrote the manuscript. DMN, JW and YLY revised the report. JJW and JXS did the statistical analysis. All authors reviewed the report and approved the article to be published.

\section{Competing interests}

The author(s) declared no potential conflicts of interest with the research, authorship, and publication of this article.

\section{Consent for publication \\ Not applicable.}

\section{Ethical approval and consent to participate}

Ethics Committee of Jinling Hospital (REC number: GH23335H) and all the subjects provided written informed consent prior to the study.

Received: 1 October 2016 Accepted: 15 December 2016

Published online: 10 January 2017

\section{References}

1. Mendivil CO, Furtado J, Morton AM, Wang L, Sacks FM. Novel pathways of apolipoprotein A-I metabolism in high-density lipoprotein of different Sizes in Humans. Arterioscler Thromb Vasc Biol. 2016;36:156-65.

2. Miida T, Obayashi K, Seino U, Zhu Y, Ito T, Kosuge K, et al. LCAT-dependent conversion rate is a determinant of plasma prebeta1-HDL concentration in healthy Japanese. Clin Chim Acta. 2004;350:107-14.

3. Rousset X, Vaisman B, Amar M, Sethi AA, Remaley AT. Lecithin: cholesterol acyltransferase-from biochemistry to role in cardiovascular disease. Curr Opin Endocrinol. 2009;16:163-71.

4. de Grooth GJ, Klerkx AH, Stroes ES, Stalenhoef AF, Kastelein JJ, Kuivenhoven JA. A review of CETP and its relation to atherosclerosis. J Lipid Res. 2004;45:1967-74.

5. de la Llera-Moya M, Drazul-Schrader D, Asztalos BF, Cuchel M, Rader DJ, Rothblat GH. The ability to promote efflux via ABCA1 determines the capacity of serum specimens with similar high-density lipoprotein cholesterol to remove cholesterol from macrophages. Arterioscler Thromb Vasc Biol. 2010;30:796-801.

6. Miida T, Sakai K, Ozaki K, Nakamura Y, Yamaguchi T, Tsuda T, et al. Bezafibrate increases prebeta 1-HDL at the expense of HDL2b in hypertriglyceridemia. Arterioscler Thromb Vasc Biol. 2000;20:2428-33.

7. Miida T, Miyazaki O, Hanyu O, Nakamura Y, Hirayama S, Narita l, et al. LCATdependent conversion of prebeta1-HDL into alpha-migrating $\mathrm{HDL}$ is severely delayed in hemodialysis patients. J Am Soc Nephrol: JASN. 2003;14:732-8.

8. Hirayama S, Miida T, Miyazaki O, Aizawa Y. Pre beta1-HDL concentration is a predictor of carotid atherosclerosis in type 2 diabetic patients. Diabetes Care. 2007;30:1289-91.

9. Filippatos TD, Liberopoulos EN, Kostapanos M, Gazi IF, Papavasiliou EC, Kiortsis DN, et al. The effects of orlistat and fenofibrate, alone or in combination, on high-density lipoprotein subfractions and pre-beta1-HDL levels in obese patients with metabolic syndrome. Diabetes Obes Metab. 2008;10:476-83.

10. Guey LT, Pullinger CR, Ishida BY, O'Connor PM, Zellner C, Francone OL, et al. Relation of increased prebeta-1 high-density lipoprotein levels to risk of coronary heart disease. Am J Cardiol. 2011;108:360-6. 
11. Chen Y, Dong J, Chen X, Jiang H, Bakillah A, Zhang X, et al. Human serum prebeta1-high density lipoprotein levels are independently and negatively associated with coronary artery diseases. Nutr Metab. 2016;13:36.

12. Tosheska K, Labudovic D, Jovanova S, Jaglikovski B, Alabakovska S. Cholestery ester transfer protein, low density lipoprotein particle size and intima media thickness in patients with coronary heart disease. Bosn J Basic Med Sci. 2011; 11:169-73.

13. Scharnagl H, Heuschneider C, Sailer S, Kleber ME, Marz W, Ritsch A. Decreased cholesterol efflux capacity in patients with low cholesteryl ester transfer protein plasma levels. Eur J Clin Invest. 2014;44:395-401.

14. Rashid S, Sniderman A, Melone M, Brown PE, Otvos JD, Mente A, et al. Elevated cholesteryl ester transfer protein (CETP) activity, a major determinant of the atherogenic dyslipidemia, and atherosclerotic cardiovascular disease in South Asians. Eur J Prev Cardiol. 2015;22:468-77.

15. Francone $\mathrm{OL}$, Royer $\mathrm{L}$, Haghpassand M. Increased prebeta-HDL levels, cholesterol efflux, and LCAT-mediated esterification in mice expressing the human cholesteryl ester transfer protein (CETP) and human apolipoprotein A-I (apoA-I) transgenes. J Lipid Res. 1996;37:1268-77.

16. Gensini GG. A more meaningful scoring system for determining the severity of coronary heart disease. Am J Cardiol. 1983;51:606.

17. Li S, Zhang Y, Xu RX, Guo YL, Zhu CG, Wu NQ, et al. Proprotein convertase subtilisin-kexin type 9 as a biomarker for the severity of coronary artery disease. Ann Med. 2015;47:386-93.

18. Miida T, Miyazaki O, Nakamura Y, Hirayama S, Hanyu O, Fukamachi I, et al. Analytical performance of a sandwich enzyme immunoassay for pre beta 1-HDL in stabilized plasma. J Lipid Res. 2003;44:645-50.

19. Wang J, Zhuang Y, Lui X, Li Y, Qiang H. Serum cholesteryl ester transfer protein concentrations in healthy Chinese subjects and cardio-cerebrovascular disease patients. Clin Chim Acta. 2001;305:19-25.

20. Wang J, Gu Q, Li K, Zhang C. CETP and oxidized LDL levels increase in dyslipidemic subjects. Clin Biochem. 2007:40:995-9.

21. Wang JJ, Gong JB, Li HQ, Niu DM, Han AZ, Wu J, et al. Lipoprotein(a) complexes with beta2-glycoprotein I in patients with coronary artery disease. J Atheroscler Thromb. 2012;19:81-9.

22. Sethi AA, Sampson M, Warnick R, Muniz N, Vaisman B, Nordestgaard BG, et al. High pre-beta1 HDL concentrations and low lecithin: cholesterol acyltransferase activities are strong positive risk markers for ischemic heart disease and independent of HDL-cholesterol. Clin Biochem. 2010;56:1128-37.

23. Tashiro J, Miyazaki O, Nakamura Y, Miyazaki A, Fukamachi I, Bujo H, et al. Plasma pre beta1-HDL level is elevated in unstable angina pectoris. Atherosclerosis. 2009;204:595-600

24. Tian L, Li C, Liu Y, Chen Y, Fu M. The value and distribution of high-density lipoprotein subclass in patients with acute coronary syndrome. PLoS One. 2014:9:e85114.

25. Miyazaki O, Kobayashi J, Fukamachi I, Miida T, Bujo H, Saito Y. A new sandwich enzyme immunoassay for measurement of plasma pre-beta1-HDL levels. J Lipid Res. 2000;41:2083-8.

26. Asztalos BF, Cupples LA, Demissie S, Horvath KV, Cox CE, Batista MC, et al. High-density lipoprotein subpopulation profile and coronary heart disease prevalence in male participants of the framingham offspring study. Arterioscl Throm Vas. 2004;24:2181-7.

27. Asztalos BF, Collins D, Cupples LA, Demissie S, Horvath KV, Bloomfield HE, et al. Value of high-density lipoprotein (HDL) subpopulations in predicting recurrent cardiovascular events in the Veterans Affairs HDL intervention trial. Arterioscl Throm Vas. 2005;25:2185-91.

28. van Tienhoven-Wind $L$, Perton FG, Dullaart RP. Pre-beta-HDL formation relates to high-normal free thyroxine in type 2 diabetes mellitus. Clin Biochem. 2016;49:41-6.

29. Kane JP, Malloy MJ. Prebeta-1 HDL and coronary heart disease. Curr Opin Lipidol. 2012;23:367-71.

30. Rohatgi A, Grundy SM. Cholesterol Efflux capacity as a therapeutic target: rationale and clinical implications. J Am Coll Cardiol. 2015;66:2211-3.

31. Nofer JR, Remaley AT. Tangier disease: still more questions than answers. Cell Mol Life Sci. 2005;62:2150-60.

32. Samyn H, Moerland M, van Haperen R, Grosveld F, van TOL A, et al. Elevation of systemic PLTP, but not macrophage-PLTP, impairs macrophage reverse cholesterol transport in transgenic mice. Atherosclerosis. 2009;204:429-34.

33. Villard EF, El Khoury P, Duchene E, Bonnefont-Rousselot D, Clement K,

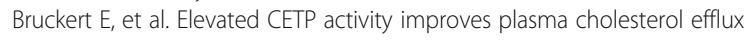
capacity from human macrophages in women. Arterioscl Thromb Vasc Biol. 2012;32:2341-9.
34. Carpentier YA, Scruel O. Changes in the concentration and composition of plasma lipoproteins during the acute phase response. Curr Opin Clin Nutr Metab Care. 2002;5:153-8.

35. Gonzalez-Pacheco H, Amezcua-Guerra LM, Vazquez-Rangel A, MartinezSanchez C, Perez-Mendez O, Verdejo J, Bojalil R. Levels of high-density lipoprotein cholesterol are associated with biomarkers of inflammation in patients with acute coronary syndrome. Am J Cardiol. 2015;116:1651-7.

36. Miida T, Ozaki K, Murakami T, Kashiwa T, Yamadera T, Tsuda T, et al. Prebeta1-high-density lipoprotein (prebeta1-HDL) concentration can change with low-density lipoprotein-cholesterol (LDL-C) concentration independent of cholesteryl ester transfer protein (CETP). Clin Chim Acta. 2000;292:69-80.

37. Kunitake ST, Mendel CM, Hennessy LK. Interconversion between apolipoprotein A-I-containing lipoproteins of pre-beta and alpha electrophoretic mobilities. J Lipid Res. 1992;33:1807-16.

38. Sugano M, Makino N, Yanaga T. Effects of hepatic HDL-related mRNAs on plasma prebeta HDL in cholesterol-fed rabbits. Artery. 1997;22:182-205.

39. Miida T, Yamaguchi T, Tsuda T, Okada M. High prebeta1-HDL levels in hypercholesterolemia are maintained by probucol but reduced by a lowcholesterol diet. Atherosclerosis. 1998;138:129-34.

40. Nicholls SJ, Ruotolo G, Brewer HB, Kane JP, Wang MD, Krueger KA, et al. Cholesterol efflux capacity and pre-beta-1 HDL concentrations are increased in dyslipidemic patients treated with evacetrapib. J Am Coll Cardiol. 2015;66: 2201-10.

41. de Vries R, Kerstens MN, Sluiter WJ, Groen AK, van Tol A, Dullaart RP. Cellular cholesterol efflux to plasma from moderately hypercholesterolaemic type 1 diabetic patients is enhanced, and is unaffected by simvastatin treatment. Diabetologia. 2005;48:1105-13.

\section{Submit your next manuscript to BioMed Central and we will help you at every step:}

- We accept pre-submission inquiries

- Our selector tool helps you to find the most relevant journal

- We provide round the clock customer support

- Convenient online submission

- Thorough peer review

- Inclusion in PubMed and all major indexing services

- Maximum visibility for your research

Submit your manuscript at www.biomedcentral.com/submit
) Biomed Central 